\title{
Estimate of prevalent ischemic stroke from triglyceride glucose-body mass index in the general population
}

Zhi Du', Liying Xing ${ }^{2}$, Min Lin ${ }^{3}$ and Yingxian Sun ${ }^{*}$ (e)

\begin{abstract}
Background: To investigate the relationship between triglyceride glucose-body mass index (TyG-BMI) and ischemic stroke.

Methods: Leveraging two Chinese general population surveys, the Northeast China Rural Cardiovascular Health Study (NCRCHS, N =11,097) and the National Stroke Screening and Intervention Program in Liaoning (NSSIPL, $N=10,862)$, we evaluated the relationship between TyG-BMI and ischemic stroke by a restricted cubic spline and multivariate logistic regression after adjusting age, sex, level of education, exercise regularly, current smoking, current drinking, atrial fibrillation, hypertension, coronary artery disease, low-density lipoprotein cholesterol, and high-density lipoprotein cholesterol. The category-free analysis was used to determine whether TyG-BMI enhanced the capacity of estimating ischemic stroke.
\end{abstract}

Results: A total of 596 and 347 subjects, respectively, from NSSIPL and NCRCHS were survivors of ischemic stroke. In NSSIPL, the relationship between TyG-BMI and ischemic stroke was linear and did not have a threshold or saturation effect according to the results of the restricted cubic spline. The regression analysis indicated that the risk of ischemic stroke increased 20\% for per SD increase of TyG-BMI after multivariate adjustment [odds ratio (OR): 1.20, 95\% confidence interval (Cl): 1.10-1.32]. Compared with those in the lowest tertile, the risk of ischemic stroke in subjects with intermediate and high TyG-BMI was significantly higher [OR (95\% Cl): 1.39 (1.10-1.74); OR (95\% Cl) 1.72 (1.37-2.17), respectively]. Category-free analysis indicated that TyG-BMI had a remarkable improvement in the ability to estimate prevalent ischemic stroke [NRI (95\% Cl): $0.188(0.105-0.270)]$. These abovementioned relationships were confirmed in NCRCHS.

Conclusions: The present study found the robust correlation between TyG-BMI and ischemic stroke, independently of a host of conventional risk factors. Meanwhile, our findings also suggested the potential usefulness of TyG-BMI to improve the risk stratification of ischemic stroke.

Keywords: Triglyceride glucose-body mass index, Ischemic stroke, Insulin resistance, Epidemiology

*Correspondence: sunyingxian_cmu@126.com

1 Department of Cardiovascular Medicine, The First Hospital of China

Medical University, Shenyang 110001, Liaoning, China

Full list of author information is available at the end of the article

\section{Background}

As the leading cause of death and disability in most countries, stroke presents an increasing burden on the global healthcare system [1]. According to the Global Burden of Disease 2013 Study, there were approximately 25.7 million stroke survivors alive, 6.5 million stroke-related deaths and 113 million stroke-related 
disability-adjusted life-years worldwide [2]. Among all patients with incident stroke who survived or died, ischemic stroke is the most common pathological type $[2,3]$. Although the age-standardized mortality rate of stroke has declined around the world, the incidence and prevalence of stroke are still explosively increasing in China [2-4]. It is generally known that smoking, hypertension, and diabetes are major risk factors for stroke $[1,4]$. Worse outcomes are ordinarily found in patients without typical risk factors for stroke [5]. Therefore, there is an urgent need to develop some cost-effective and reproducible markers to improve stroke risk stratification, especially in ischemic stroke.

Insulin resistance (IR) is a frequent pathological condition in which cells have an impairment in the ability to respond to the hormone insulin. Accumulating evidence has revealed a significantly positive association between IR and ischemic stroke. Experimental studies have partially identified these mechanisms: atherosclerosis is a notoriously pathophysiological process of ischemic stroke, IR can accelerate the progression of atherosclerosis by decreasing eNOS activation (triggering endothelium dysfunction), increasing VCAM-1 expression (triggering leukocyte adhesion) in endothelial cells and migration and proliferation of vascular smooth muscle cells $[6,7]$. Meanwhile, IR can potentially induce prolonged endoplasmic reticulum stress in macrophages and contribute to macrophage apoptosis, which subsequently results in plaque necrosis in advanced atherosclerosis [6, 8]. Epidemiological evidence also supports the association between IR and ischemic stroke [9-12]. Clinical trials have also suggested the effectiveness of insulin and insulin sensitizers in the treatment of ischemic stroke [13, 14]. Hence, early identification and early intervention of IR are of great clinical significance in reducing the risk of ischemic stroke. The current gold standard for diagnosing IR is the euglycemic-hyperinsulinemic clamp, but its application is confined by the complicated procedure, high cost, and ethical concerns [15].

Recently, Yu-Lin Ko has put forward a novel index named the Triglyceride Glucose-Body Mass Index (TyG-BMI), calculated as Ln [TG (mg/dl) *FBG (mg/ $\mathrm{dl}) / 2]^{*}$ BMI $\left(\mathrm{kg} / \mathrm{m}^{2}\right)$, as a potential and straightforward marker for IR [16]. Previous studies have evaluated its usefulness to multiple IR-related clinical diseases [1721]. Nevertheless, it remains unclear whether TyG-BMI can identify ischemic stroke, independently of other conventional risk factors at present. Accordingly, the purpose of the present study was to assess whether a higher level of TyG-BMI was associated with ischemic stroke in two surveys of two population-based samples in China.

\section{Methods}

\section{Study participants}

Northeast China Rural Cardiovascular Health Study (NCRCHS) was a population-based cross-sectional investigation, and the design, survey methods, and laboratory techniques have been described in previous studies [22, 23]. Using the method of multi-stage, stratified and cluster random sampling, 14,016 permanent residents in Dawa, Zhangwu and Liaoyang counties in Liaoning province were invited to participate in the study, and a total of 11,597 subjects aged $\geq 35$ years completed the study from January 2013 to August 2013. In the present study, 482 participants were furtherly excluded for the reasons: lacking or unreadable electrocardiograms (ECGs, $\mathrm{n}=238)$, and missing data $(\mathrm{n}=244)$. Eventually, 11,097 (95.6\%) subjects were analyzed. The study was approved by the Ethics Committee of China Medical University (Shenyang, China). Written informed consent was obtained from all participants.

The National Stroke Screening and Intervention Program in Liaoning (NSSIPL) was a cross-sectional study conducted in rural areas of Liaoning province from September 2017 to May 2018. The design and enrolment process of the study has been previously described [24, 25]. Briefly, the method of multi-stage, stratified, and cluster random sampling was adopted to randomly select four counties, including Liaoyang, Chaoyang, Donggang, and Lingyuan, from the central, eastern and western regions of Liaoning province, and 19 villages were involved. With the exception of those who were pregnant or had mental disorders, all permanent residents aged at least 40 in each village $(n=12,808)$ were eligible to participate, and a total of $10,926(85.3 \%)$ completed the survey. Sixtyfour participants were furtherly excluded for the following reasons: the absence of blood samples $(n=26)$ and abnormal or missing information $(n=38)$. Finally, data of $10,862(99.4 \%)$ subjects were analyzed. The study was approved by the Central Ethics Committee at the China National Center for Cardiovascular Disease and received the written informed consent of all participants.

\section{Data collection and measurement}

Data collection and anthropometric measurements were similar in NCRCHS and NSSIPL. Demographic and clinical information, including age, sex, socioeconomic status, lifestyle (smoking, drinking, and physical activity) were collected by a team of cardiologists, neuroscientists, and experts trained in chronic disease prevention and control. Self-administered questionnaires were fulfilled by face-to-face interviews during a clinical visit. Before the start of data collection, all team members were selected through a rigorous process, and pilot interviews with volunteers were completed. 
If subjects answered "yes" to the question that "Have you been diagnosed with [specific disease] by a registered physician?", then they were identified as having complications including hypertension, dyslipidemia, diabetes, and atrial fibrillation or a history of a specific disease such as diabetes, coronary heart disease, (CHD) or cerebrovascular disease. Subsequently, the subjects with self-reported complications and specific diseases were asked if they had taken prescription drugs to control the disease in the past 2 weeks. Those who answered "yes" were asked to report the name, dose, and frequency of each drug if known. Those who did not remember the exact dose were asked the number of pills taken.

Physical measurements were obtained, including height and weight. The weight and height were measured to the nearest $0.1 \mathrm{~kg}$ and $0.1 \mathrm{~cm}$, respectively, with participants wearing light clothing and no shoes. The body mass index (BMI) was defined as weight in kilograms divided by the square of the height in meter. After sitting for at least $5 \mathrm{~min}$, each participant was measured blood pressure three times at 2 min intervals with a standardized, automated electronic sphygmomanometer (HEM907; Omron, Tokyo, Japan in NCRCHS; J30; Omron, Kyoto, Japan in NSSIPL). A central steering committee with a subcommittee for quality control was established to ensure that data were obtained according to standardized protocols.

Blood samples were taken from participants who fasted for at least $8 \mathrm{~h}$. The samples were taken from an antecubital vein using EDTA (BD Vacutainer tubes containing ethylenediaminetetraacetic acid; Becton, Dickinson and Co., Franklin Lakes, NJ, USA), and serum was subsequently isolated from the whole blood. Thereafter, the serum samples were cryopreserved at $-20{ }^{\circ} \mathrm{C}$. Subsequently, the biochemical parameters including fasting blood glucose (FBG), glycosylated hemoglobin (HbA1c), triglyceride (TG), total cholesterol (TC), low-density lipoprotein cholesterol (LDL-C), and high-density lipoprotein cholesterol (HDL-C) were measured by an Olympus AU640 Auto-Analyzer (Olympus Corp., Kobe, Japan) in NCRCHS and an Abbott Diagnostics C800i autoanalyzer (Abbott Laboratories, Abbott Park, IL, USA) in NSSIPL, respectively. Because Laboratory tests were conducted in three laboratories in NSSIPL, we randomly selected $10 \%$ samples from each laboratory for centralized retesting by China's Ministry of Health's National Center for Clinical Laboratories to ensure the accuracy of the test.

\section{Definition}

TyG-BMI was calculated as follows: TyG-BMI $=\mathrm{Ln}$ [TG $\quad(\mathrm{mg} / \mathrm{dl}) *$ FBG $\quad(\mathrm{mg} / \mathrm{dl}) / 2]$ *BMI $\quad\left(\mathrm{kg} / \mathrm{m}^{2}\right) \quad[16]$. Ischemic stroke was diagnosed by a neurologist on the recommendation of the World Health Organization and confirmed by computed tomography (CT) and/ or magnetic resonance imaging (MRI) [26]. Hypertension was determined as mean systolic blood pressure $(\mathrm{SBP}) \geq 140 \mathrm{mmHg}$ or mean diastolic blood pressure (DBP) $\geq 90 \mathrm{mmHg}$ and/or self-reported use of antihypertensive drugs in the past 2 weeks [27]. Dyslipidemia was diagnosed if the participants met one of the following criteria: (1) serum TC level $\geq 6.22 \mathrm{mmol} / \mathrm{L}$; (2) serum LDL-C level $\geq 4.14 \mathrm{mmol} / \mathrm{L}$; (3) serum TG level $\geq 2.26 \mathrm{mmol} / \mathrm{L}$; (4) serum HDL-C level $<1.04 \mathrm{mmol} / \mathrm{L}$; and (5) selfreported use of lipid-regulating drugs [28]. Diabetes mellitus was defined as $\mathrm{FBG} \geq 7.0 \mathrm{mmol} / \mathrm{L}$ or $\mathrm{HbA} 1 \mathrm{c} \geq 6.5 \%$, and/or self-reported diabetes [29]. AF was diagnosed by a physician based on an electrocardiogram and/or previous diagnosis. Regular exercise was defined as moderate-intensity exercise (equivalent to walking) $\geq 30 \mathrm{~min}$ and $\geq 3$ times per week.

\section{Statistical analysis}

Level of TyG-BMI was divided into three categories (low, intermediate, and high) by tertiles in NSSIPL. Then, according to the categories of TyG-BMI, continuous variables were presented as means and standard deviations and categorical variables were reported as frequencies and percentages in each subgroup. One way analysis of variance (ANOVA) and chi-squared tests were used, as appropriate, to compare differences among the 3 groups. The dose-response relationship between TyG-BMI and the risk of ischemic stroke was evaluated by a restricted cubic spline. Meanwhile, multivariate logistic regression analysis was carried out to evaluate the association between TyG-BMI and ischemic stroke. As a categorical variable, or a continuous variable using the standard deviation transformed, level of TyG-BMI was incorporated separately into regression model analysis, adjusting for conventional risk factors, including age, sex, level of education, exercise regularity, current smoking, current drinking, $\mathrm{AF}$, hypertension, coronary artery disease, LDL-C, and HDL-C. Odds ratios (ORs) and 95\% confidence intervals (CIs) were presented for the logistic regression analyses. TyG-BMI was derived from FBG, TG, and BMI. Therefore, to examine whether TyG-BMI was indeed an independent risk factor and whether the association between TyG-BMI and ischemic stroke depended entirely on diabetes, obesity or overweight, dyslipidemia. We additionally adjusted for diabetes, obesity or overweight, and dyslipidemia separately or simultaneously. To evaluate the extent to which TyG-BMI improved prediction performance over conventional risk factors, net reclassification improvement (NRI) was calculated and compared in NSSIPL (conventional risk factors only vs. conventional risk factors + TyG-BMI). 
Taking the NSSIPL as an example, the NCRCHS data was analyzed using the same methods to verify the conclusion of the NSSIPL. Statistical analyses were performed using SPSS software version 22.0 (SPSS Inc., Chicago, IL, USA), EmpowerStats (https://www.empowersta ts.com, X\&Y Solutions, Inc., Boston, MA) and statistical software packages $\mathrm{R}$ (https://www.R-project.org, The $\mathrm{R}$ Foundation); $p$ values $<0.05$ were considered statistically significant.

\section{Results}

Our study included 10,862 NSSIPL subjects (mean age: $59.95 \pm 10.06$, males: $40.2 \%$ ) and 11,097 NCRCHS participants (mean age: $53.83 \pm 10.56$, males: $45.6 \%$ ). Clinical characteristics by level of TyG-BMI in two surveys were shown in Tables 1 and 2, respectively. The average TyGBMI level of individuals in the two cross-sections was analogous (means \pm standard deviations: $218.24 \pm 40.83$ in NSSIPL; $216.74 \pm 40.17$ in NCRCHS). 596 of the 10,896 NSSIPL subjects (5.4\%) and 347 of the 11,097
NCRCHS participants (3.1\%) were survivors of ischemic stroke. Compared with subjects with lower levels of TyG-BMI, individuals with higher TyG-BMI had significantly higher SBP, DBP, FBG, TG, TC, LDL-C, and lower HDL-C in both NSSIPL and NCRCHS (all $p s<0.05$ ). In addition, subjects with higher levels of TyG-BMI had a significantly higher prevalence of overweight or obesity, diabetes, dyslipidemia, CHD, and ischemic stroke (all $p s<0.05$ ).

The linear associations were demonstrated between the level of TyG-BMI and ischemic stroke in two surveys (Fig. 1). The risk of ischemic stroke obviously increased with the level of TyG-BMI. Further, the relationships were assessed separately by the multivariate logistic regression models in both surveys, and results were shown in Table 3. In NSSIPL, the regression analysis using the standard deviation transformed TyG-BMI as a continuous variable indicated that the risk of ischemic stroke increased $20 \%$ per SD increase after multivariate adjustment [OR: 1.20, 95\% CI: 1.10-1.32]. Then, as

Table 1 Baseline characteristics of the NSSIPL participants according to TyG-BMI levels

\begin{tabular}{|c|c|c|c|c|}
\hline \multirow[t]{2}{*}{ Characteristics } & \multicolumn{3}{|c|}{ Level of TyG-BMI } & \multirow[b]{2}{*}{$\begin{array}{l}p \text { value } \\
\text { for liner } \\
\text { trend }\end{array}$} \\
\hline & $\begin{array}{l}\text { Low } \\
(N=3617)\end{array}$ & Intermediated $(N=3628)$ & $\begin{array}{l}\text { High } \\
(N=3617)\end{array}$ & \\
\hline Age (years) & $61.64 \pm 10.82$ & $59.63 \pm 9.81$ & $58.57 \pm 9.26$ & $<0.001$ \\
\hline Male $[\mathrm{n}(\%)]$ & 1768 (48.9) & $1381(38.1)$ & $1215(33.6)$ & $<0.01$ \\
\hline Education [n (\%)] & & & & $<0.001$ \\
\hline Primary school or below & $2209(61.1)$ & $2135(58.8)$ & $2080(57.5)$ & \\
\hline Middle school & $1140(31.5)$ & $1165(32.1)$ & 1169 (32.3) & \\
\hline High school or higher & $268(7.4)$ & $328(9.0)$ & $368(10.2)$ & \\
\hline Current smoking [n (\%)] & $1305(36.1)$ & $881(24.3)$ & $687(19.0)$ & $<0.001$ \\
\hline Current drinking [n (\%)] & $1188(32.8)$ & 969 (26.7) & $900(24.9)$ & $<0.001$ \\
\hline Regular exercise [n (\%)] & $2979(82.4)$ & $3089(85.1)$ & $2997(82.9)$ & 0.569 \\
\hline $\mathrm{SBP}(\mathrm{mmHg})$ & $139.50 \pm 23.24$ & $145.70 \pm 22.47$ & $151.88 \pm 23.50$ & $<0.001$ \\
\hline $\mathrm{DBP}(\mathrm{mmHg})$ & $83.31 \pm 11.57$ & $86.45 \pm 11.28$ & $90.28 \pm 11.88$ & $<0.001$ \\
\hline FBG (mmol/L) & $5.64 \pm 1.16$ & $6.06 \pm 1.59$ & $6.78 \pm 2.42$ & $<0.001$ \\
\hline $\mathrm{TG}(\mathrm{mmol} / \mathrm{L})$ & $0.98 \pm 0.45$ & $1.50 \pm 0.89$ & $2.48 \pm 2.15$ & $<0.001$ \\
\hline $\mathrm{TC}(\mathrm{mmol} / \mathrm{L})$ & $4.79 \pm 0.98$ & $5.14 \pm 1.09$ & $5.41 \pm 1.20$ & $<0.001$ \\
\hline LDL-C (mmol/L) & $2.11 \pm 0.80$ & $2.37 \pm 1.00$ & $2.58 \pm 1.18$ & $<0.001$ \\
\hline $\mathrm{HDL}-\mathrm{C}(\mathrm{mmol} / \mathrm{L})$ & $2.07 \pm 0.61$ & $1.94 \pm 0.74$ & $1.86 \pm 0.88$ & $<0.001$ \\
\hline Obesity/overweight [n (\%)] & $26(0.7)$ & $1542(42.5)$ & 3397 (93.9) & $<0.001$ \\
\hline Hypertension [n (\%)] & $1757(48.6)$ & $2185(60.2)$ & $2636(72.9)$ & $<0.001$ \\
\hline Diabetes [n (\%)] & $217(6.0)$ & $508(14.0)$ & $1033(28.6)$ & $<0.001$ \\
\hline Dyslipidemia [n (\%)] & $422(11.7)$ & $998(27.5)$ & $1946(53.8)$ & $<0.001$ \\
\hline Atrial fibrillation [n (\%)] & $49(1.4)$ & $33(0.9)$ & $35(1.0)$ & 0.111 \\
\hline $\mathrm{CHD}[\mathrm{n}(\%)]$ & $123(3.4)$ & 135 (3.7) & $197(5.4)$ & $<0.001$ \\
\hline Ischemic stroke [n (\%)] & $159(4.4)$ & $194(5.3)$ & $243(6.7)$ & $<0.001$ \\
\hline
\end{tabular}

NSSIPL National Stroke Screening and Intervention Program in Liaoning, TyG-BMI triglyceride glucose-body mass index, SBP systolic blood pressure, DBP diastolic blood pressure, FBG fasting blood glucose, TG triglycerides, TC total cholesterol, $L D L-C$ low-density lipoprotein cholesterol, $H D L-C$ high-density lipoprotein cholesterol, CHD coronary heart disease 
Table 2 Baseline characteristics of the NCRCHS participants according to TyG-BMI levels

\begin{tabular}{|c|c|c|c|c|}
\hline \multirow[t]{2}{*}{ Characteristics } & \multicolumn{3}{|l|}{ Level of TyG-BMI } & \multirow[b]{2}{*}{$\begin{array}{l}p \text { value } \\
\text { for liner } \\
\text { trend }\end{array}$} \\
\hline & Low $(N=3695)$ & Intermediated $(N=3708)$ & High $(N=3695)$ & \\
\hline Age (years) & $53.79 \pm 11.18$ & $53.81 \pm 10.49$ & $53.89 \pm 9.99$ & 0.677 \\
\hline Male $[n(\%)]$ & $1738(47.0)$ & $1669(45.0)$ & $1654(44.8)$ & 0.050 \\
\hline Education [n (\%)] & & & & 0.522 \\
\hline Primary school or below & $1802(48.8)$ & $1869(50.4)$ & $1876(50.8)$ & \\
\hline Middle school & $1578(42.7)$ & $1476(39.8)$ & $1466(39.7)$ & \\
\hline High school or higher & $315(8.5)$ & $362(9.8)$ & $353(9.5)$ & \\
\hline Current smoking [n (\%)] & $1532(41.5)$ & $1229(33.2)$ & $1134(30.7)$ & $<0.001$ \\
\hline Current drinking [n (\%)] & $833(22.5)$ & $831(22.4)$ & $806(21.8)$ & 0.450 \\
\hline Regular exercise [n (\%)] & $2676(72.4)$ & $2668(72.0)$ & $2471(66.9)$ & $<0.001$ \\
\hline $\mathrm{SBP}(\mathrm{mmHg})$ & $134.54 \pm 21.50$ & $141.71 \pm 23.01$ & $148.81 \pm 23.57$ & $<0.001$ \\
\hline $\mathrm{DBP}(\mathrm{mmHg})$ & $78.09 \pm 10.80$ & $81.94 \pm 11.31$ & $85.98 \pm 11.82$ & $<0.001$ \\
\hline $\mathrm{FBG}(\mathrm{mmol} / \mathrm{L})$ & $5.49 \pm 0.93$ & $5.77 \pm 1.32$ & $6.45 \pm 2.20$ & $<0.001$ \\
\hline $\mathrm{TG}(\mathrm{mmol} / \mathrm{L})$ & $0.97 \pm 0.44$ & $1.40 \pm 0.80$ & $2.55 \pm 2.15$ & $<0.001$ \\
\hline $\mathrm{TC}(\mathrm{mmol} / \mathrm{L})$ & $4.93 \pm 0.97$ & $5.22 \pm 1.03$ & $5.55 \pm 1.16$ & $<0.001$ \\
\hline LDL-C (mmol/L) & $2.64 \pm 0.71$ & $2.95 \pm 0.78$ & $3.18 \pm 0.88$ & $<0.001$ \\
\hline $\mathrm{HDL}-\mathrm{C}(\mathrm{mmol} / \mathrm{L})$ & $1.55 \pm 0.41$ & $1.42 \pm 0.37$ & $1.26 \pm 0.29$ & $<0.001$ \\
\hline Obesity/overweight [n (\%)] & $26(0.7)$ & $1470(39.7)$ & 3471 (93.9) & $<0.001$ \\
\hline Hypertension [n (\%)] & 1325 (35.9) & $1884(50.8)$ & $2449(66.3)$ & $<0.001$ \\
\hline Diabetes [n (\%)] & $128(3.5)$ & $272(7.3)$ & $752(20.4)$ & $<0.001$ \\
\hline Dyslipidemia [n (\%)] & $674(18.2)$ & 1315 (35.5) & $2406(65.1)$ & $<0.001$ \\
\hline Atrial fibrillation [n (\%)] & $42(1.1)$ & $43(1.2)$ & $53(1.4)$ & 0.248 \\
\hline $\mathrm{CHD}[\mathrm{n}(\%)]$ & $144(3.9)$ & $171(4.6)$ & $258(7.0)$ & $<0.001$ \\
\hline Ischemic stroke [n (\%)] & $68(1.8)$ & $99(2.7)$ & $180(4.9)$ & $<0.001$ \\
\hline
\end{tabular}

Abbreviations as in Table 1; NCRCHS Northeast China Rural Cardiovascular Health Study
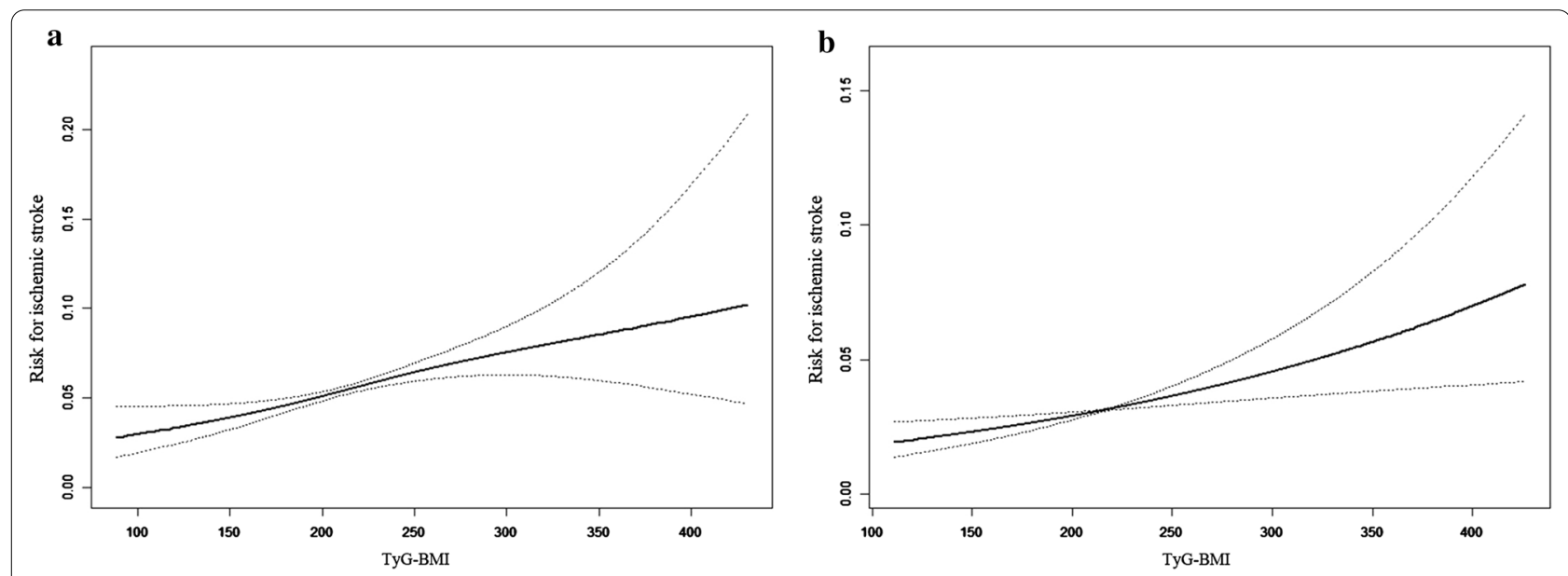

Fig. 1 The associated between TyG-BMI and ischemic stroke in NSSIPL (a) and NCRCHS (b) by restricted cubic spline

a categorical variable (low vs. intermediate vs. high) brought into the regression equation, TyG-BMI implied a similar correlation with ischemic stroke. Participants with low levels of TyG-BMI were used as a reference, and the risk of ischemic stroke in subjects with intermediate and high TyG-BMI was significantly higher [OR (95\% CI): 
Table 3 The association between TyG-BMI and ischemic stroke by multivariate logistic regression analysis

\begin{tabular}{|c|c|c|c|c|c|c|c|c|}
\hline \multirow[t]{2}{*}{ TyG-BMI } & \multicolumn{8}{|l|}{ Sensitive analysis } \\
\hline & Mode 1 OR $(95 \% \mathrm{Cl})$ & $P$ value & Mode 2 OR $(95 \% \mathrm{Cl})$ & $P$ value & Mode 3 OR $(95 \% \mathrm{Cl})$ & $P$ value & Mode 4 OR $(95 \% \mathrm{Cl})$ & $P$ value \\
\hline \multicolumn{9}{|l|}{ NSSIPL $(N=10,862)$} \\
\hline \multicolumn{9}{|l|}{ Continuous } \\
\hline Per SD increase & $1.20(1.10-1.32)$ & $<0.001$ & $1.16(1.06-1.27)$ & 0.002 & $1.20(1.07-1.36)$ & 0.003 & $1.07(0.93-1.23)$ & 0.324 \\
\hline \multicolumn{9}{|l|}{ Categorical } \\
\hline Low & 1 & - & 1 & - & 1 & - & 1 & - \\
\hline Intermediated & $1.39(1.10-1.74)$ & 0.005 & $1.34(1.07-1.69)$ & 0.012 & $1.44(1.12-1.85)$ & 0.004 & $1.30(1.01-1.68)$ & 0.044 \\
\hline High & $1.72(1.37-2.17)$ & $<0.001$ & $1.58(1.25-2.01)$ & $<0.001$ & $1.89(1.35-2.64)$ & $<0.001$ & $1.48(1.03-2.13)$ & 0.033 \\
\hline \multicolumn{9}{|c|}{$\operatorname{NCRCHS}(N=11,097)$} \\
\hline \multicolumn{9}{|l|}{ Continuous } \\
\hline Per SD increase & $1.21(1.07-1.36)$ & 0.002 & $1.17(1.03-1.33)$ & 0.015 & $1.25(1.06-1.48)$ & 0.008 & $1.13(0.96-1.35)$ & 0.170 \\
\hline \multicolumn{9}{|l|}{ Categorical } \\
\hline Low & 1 & - & 1 & - & 1 & - & 1 & - \\
\hline Intermediated & $1.22(0.88-1.71)$ & 0.240 & $1.21(0.87-1.69)$ & 0.262 & $1.37(0.97-1.95)$ & 0.076 & $1.29(0.91-1.83)$ & 0.152 \\
\hline High & $1.84(1.32-2.56)$ & $<0.001$ & $1.75(1.25-2.45)$ & 0.001 & $2.58(1.62-4.13)$ & $<0.001$ & $2.12(1.32-3.41)$ & 0.002 \\
\hline \multicolumn{9}{|c|}{ Model 1: Adjusting for age, sex, education, regular exercise, current smoking, current drinking, atrial fibrillation, hypertension, coronary heart disease, LDL-C, HDL-C } \\
\hline \multicolumn{9}{|c|}{ Model 2: Adjusting for all risk the factors in model 1 and diabetes } \\
\hline \multicolumn{9}{|c|}{ Model 3: Adjusting for all risk the factors in model 1 and overweight or obesity } \\
\hline \multicolumn{9}{|c|}{$\begin{array}{l}\text { Model 4: Adjusting for age, sex, education, regular exercise, current smoking, current drinking, atrial fibrillation, hypertension, coronary heart disease, overweight or } \\
\text { obesity and dyslipidemia }\end{array}$} \\
\hline
\end{tabular}

1.39 (1.10-1.74) in the intermediate TyG-BMI group; OR (95\% CI) 1.72 (1.37-2.17) in the high TyG-BMI group]. The linkage between TyG-BMI and ischemic stroke did not change after additionally adjusting for diabetes (OR: $1.34, p=0.012$; OR: $1.58, p<0.001)$ or obesity or overweight (OR: 1.44, $p=0.004$; OR: $1.89, p<0.001$ ). Even if we adjusted diabetes, obesity or overweight, and dyslipidemia simultaneously, the correlation between TyG-BMI and stroke still existed in the high-level group (OR: 1.30, $p=0.044$; OR: $1.48, p=0.033)$. These conclusions were confirmed among participants from NCRCHS.

The category-free analysis was performed to evaluate the effect that TyG-BMI improved the risk stratification of ischemic stroke in both NSSIPL and NCRCHS (Table 4). Compared with conventional risk factors only, analysis results showed that the ability to predict ischemic stroke was remarkably improved after adding
TyG-BMI [NRI (95\% CI): 0.188 (0.105-0.270) in NSSIPL; 0.197 (0.091-0.304) in NCRCHS].

\section{Discussion}

Our study demonstrated that a significantly positive association existed between TyG-BMI and ischemic stroke in two surveys based on the general population. The relationship was linear and independent of a host of conventional risk factors. We also noticed that the effect of TyG-BMI on ischemic stroke was exerted beyond diabetes, obesity, and dyslipidemia, although TyG-BMI was calculated from FBG, TG, and BMI. Meanwhile, our results suggested that TyG-BMI significantly improved the risk stratification of ischemic stroke. In brief, the results of our research provided clinical guidance that TyG-BMI was a potential marker to estimate prevalent ischemic stroke.

Table 4 The value that TyG-BMI improved risk stratification of ischemic stroke according to NRI

\begin{tabular}{|c|c|c|c|c|}
\hline Variable & NSSIPL $(N=10,862)$ & $P$ value & NCRCHS $(N=11,098)$ & $P$ value \\
\hline Conventional risk factors & reference & & Reference & \\
\hline Conventional risk factors + TyG-BMI & $0.188(0.105-0.270)$ & $<0.001$ & $0197(0.091-0.304)$ & $<0.001$ \\
\hline
\end{tabular}

Conventional risk factors include age, sex, education, regular exercise, current smoking, current drinking, atrial fibrillation, hypertension, coronary heart disease, LDLC, HDL-C

TyG-BMI triglyceride glucose-body mass index, NRI net reclassification improvement, NSSIPL National Stroke Screening and Intervention Program in Liaoning, NCRCHS Northeast China Rural Cardiovascular Health Study 
A previous study has proved that high levels of free fatty acids in plasma can rapidly give rise to IR in humans [30]. Meanwhile, under high-glucose conditions, glucose molecules conjugate to proteins similar to the insulin receptor on the surface of the cytoplasmic membrane, then convert to advanced glycation endproducts promptly [31]. Serum insulin cannot fit perfectly with the advanced glycation endproducts deposited on the insulin receptor and then fail to mediate insulin's stimulation of glucose transport, triggering IR [32, 33]. Recently, some studies have suggested that the product of triglycerides and glucose in plasma (TyG) is effective in detecting IR [34, 35]. Besides, BMI is a simple anthropometric parameter, which is often used as an index of obesity and IR. In obese individuals, adipose tissue lipolysis increases and releases considerable free fatty acids, which is perhaps the most paramount element in modulating insulin sensitivity $[36,37]$. Therefore, there is a reasonable hypothesis that TyG-BMI, based on the anthropometric BMI and TyG parameters, also serve as markers for IR.

In fact, the correlation between TyG-BMI and homeostatic model assessment (HOMA)-IB has been proven [16]. Subsequently, TyG-BMI was recommended as a marker to evaluate IR and IR-related diseases in many studies. A cross-sectional study conducted in rural Beijing, China confirmed that TyG-BMI was a better indicator in detecting IR [38]. The 2015 Health, Well-Being, and Aging Study also suggested that TyG-BMI could be used to assess prediabetes, although it was not the optimal index [17]. Results from a large cross-sectional survey involving 11,149 participants, the Korean National Health and Nutrition Examination Survey, indicated that TyG-BMI was an alternative marker for evaluating IR after comparing with other parameters of IR [39]. Besides, these associations between TyG-BMI with prehypertension and hypertension have been confirmed. $[20,21]$ However, the relationship between TyG-BMI and ischemic stroke remains unclear. To obtain the accurate association, other conventional risk factors including age, sex, level of education, exercise regularity, current smoking, current drinking, AF, hypertension, coronary artery disease, LDL-C, and HDL-C were thoroughly adjusted in our analysis, and results indicated that high levels of TyG-BMI was a risk factor for ischemic stroke. The relationship was linear and did not have a threshold or saturation effect according to the results of the restricted cubic spline. TyG-BMI was calculated by FBG, TG, and BMI, but the observed relationship between TyG-BMI and ischemic stroke was unlikely to be confounded by associated clinical diseases because diabetes, obesity or overweight, and dyslipidemia were adjusted in the additional analysis at the same time. This also implied that
TyG-BMI might be an alternative indicator of IR to predict ischemic stroke independently.

When adding new markers to existing prediction models, it is necessary to calculate NRI to determine whether the new assessment model is meaningful [40]. Fortunately, category-free analysis inferred that this significantly enhanced capacity of estimating ischemic stroke when adding TyG-BMI to conventional risk factors in the two investigations. The NRI value suggested that the model after addition of TyG-BMI led to a significant improvement in the risk stratification of ischemic stroke.

Our study provided the reference for the application of TyG-BMI as a clinically useful marker in identification of individual at high risk for cardiovascular disease. Tyg-BMI was calculated by FBG, TG and BMI. Obviously, these biochemical parameters can be obtained from a single sample at the same time, which is cheap and convenient compared to euglycemic-hyperinsulinemic clamp. Besides, in terms of generality, height, weight, FBG and TG are routinely performed biochemical tests in primary hospitals, which is advantage for clinical and epidemiological studies. Hence, we have reason to believe that Tyg-BMI has good application prospects to identify patients with high risk of cardiovascular disease.

The prevalence of ischemic stroke in our populations performed "significantly above" that of the 2014 national level in participants aged 40 and older (3.1\% vs. $1.8 \%$ or $5.4 \%$ vs. $1.8 \%$ ) [4]. However, this was reasonable due to our special samples, which were composed of rural population aged $\geq 40$ years in northeast China. Previous research demonstrated that stroke distributed in a northto-south gradient in China and the northern region suffered from the highest stroke burden. Simultaneously, the prevalence of stroke in rural residents was significantly higher than that in urban residents [3]. Although participants in both NSSIPL and NCRCHS hailed from northeast China, the individuals in NSSIPL were older and had higher prevalence of hypertension and diabetes. As for age, Michael et al. [41] asserted that increased risk for stroke rose more than doubles for each successive 10 years after the age of 55 . Hypertension and diabetes had invariably been well-documented risk factors for ischemic stroke [4]. Additionally, the prevalence of stroke presented an escalating trend in China, and NCRCHS was conducted 5 years ahead of NSSIPL [3]. To sum up, it was accepted that the prevalence of ischemic stroke in NSSIPL was higher than that in NCRCHS.

The present study indicated the linear association of TyG-BMI with ischemic stroke. The strengths of our results were repetitive in two surveys. However, there were some limitations in our study. Firstly, the crosssectional design of the study only allowed assessment of the associations between TyG-BMI and ischemic stroke 
rather than causal links. Secondly, although our studies contained a large number of individuals, they were from northeast China, over 40 years old, and mostly postmenopausal women. This might reduce the ability of TyGBMI to estimate ischemic stroke and restrict applicability of our results to other populations. Thirdly, the comorbidities and medical history of participants were selfreported in our study, which might lead to false-positive results. Subsequently, some confounding factors proved in other studies, such as inflammation, were not collected in our study. Finally, recruiting only ischemic stroke survivors might affect the accuracy of our results.

\section{Conclusions}

Our results suggested the independent association between TyG-BMI and ischemic stroke in the general population and the relationship was linear and did not have a threshold or saturation effect. Meanwhile, our study suggests the potential usefulness of TyG-BMI to improve the risk stratification of ischemic stroke.

\begin{abstract}
Abbreviations
BMI: Body mass index; CHD: Coronary heart disease; DAG: Diacylglycerol; DBP: Diastolic blood pressure; FBG: Fasting blood glucose; $\mathrm{HbA1c}$ : Glycosylated hemoglobin; HDL-C: High-density lipoprotein cholesterol; IR: Insulin resistance; LDL-C: Low-density lipoprotein cholesterol; NCRCHS: Northeast China Rural Cardiovascular Health Study; NSSIPL: The National Stroke Screening and Intervention Program in Liaoning; PKCE: Protein kinase C epsilon; SBP: Systolic blood pressure; TC: Total cholesterol; TG: Triglyceride; TyG-BMI:Triglyceride glucose-body mass index.
\end{abstract}

\section{Acknowledgements}

We would like to thank the CDC of Chaoyang, Lingyuan, Liaoyang, Dandong and Donggang for their assistance with this study.

\section{Authors' contributions}

ZD takes responsibility for all aspects of the reliability and freedom from bias of the data presented and their discussed interpretation. LX, SY take responsibility for research design and data collection. ZD, ML, LX, take responsibility for data collection. All authors read and approved the final manuscript.

\section{Funding}

This study was supported by the National Key R\&D Program of China (Grant No. 2017YFC1310902) and Liaoning science and technology project, Research and demonstration of hypertension diagnosis and treatment and optimization of process management in Liaoning Province community (Grant No. 2017107001). These funding bodies had no role in the study design; collection, analysis, and interpretation of data; writing of the report; or decision to submit the article for publication.

\section{Availability of data and materials}

The datasets used and/or analysed during the current study are de-identified and available from the corresponding author on reasonable request.

\section{Ethics approval and consent to participate}

The study was granted approval by the Central Ethics Committee at the China National Center for Cardiovascular Disease. Written informed consent from all participants was obtained.

\section{Consent for publication}

Not applicable.

\section{Competing interests}

The authors declare that they have no competing interests.

\section{Author details}

${ }^{1}$ Department of Cardiovascular Medicine, The First Hospital of China Medical University, Shenyang 110001, Liaoning, China. ${ }^{2}$ Disease Control and Prevention of Liaoning Province, Shenyang, Liaoning, People's Republic of China.

${ }^{3}$ Department of Cardiovascular Medicine, Benxi Central Hospital, Benxi, Liaoning, China.

Received: 12 March 2020 Accepted: 5 November 2020

Published online: 12 November 2020

\section{References}

1. O'Donnell MJ, Chin SL, Rangarajan S, Xavier D, Liu L, Zhang H, RaoMelacini P, Zhang X, Pais P, Agapay S, et al. Global and regional effects of potentially modifiable risk factors associated with acute stroke in 32 countries (INTERSTROKE): a case-control study. Lancet. 2016;388(10046):761-75.

2. Feigin VL, Krishnamurthi RV, Parmar P, Norrving B, Mensah GA, Bennett DA, Barker-Collo S, Moran AE, Sacco RL, Truelsen T, et al. Update on the global burden of ischemic and hemorrhagic stroke in 1990-2013: the GBD 2013 Study. Neuroepidemiology. 2015;45(3):161-76.

3. Wang W, Jiang B, Sun H, Ru X, Sun D, Wang L, Wang L, Jiang Y, Li Y, Wang Y, et al. Prevalence, incidence, and mortality of stroke in China: results from a nationwide population-based survey of 480,687 adults. Circulation. 2017;135(8):759-71.

4. Guan T, Ma J, Li M, Xue T, Lan Z, Guo J, Shen Y, Chao B, Tian G, Zhang Q, et al. Rapid transitions in the epidemiology of stroke and its risk factors in China from 2002 to 2013. Neurology. 2017;89(1):53-61.

5. Assmann G, Schulte H, Cullen P, Seedorf U. Assessing risk of myocardial infarction and stroke: new data from the prospective cardiovascular munster (PROCAM) study. Eur J Clin Investig. 2007;37(12):925-32.

6. Bornfeldt KE, Tabas I. Insulin resistance, hyperglycemia, and atherosclerosis. Cell Metab. 2011;14(5):575-85.

7. Deng $X L$, Liu Z, Wang C, Li Y, Cai Z. Insulin resistance in ischemic stroke. Metab Brain Dis. 2017;32(5):1323-34.

8. Moore KJ, Tabas I. Macrophages in the pathogenesis of atherosclerosis. Cell. 2011;145(3):341-55.

9. Rundek T, Gardener H, Xu Q, Goldberg RB, Wright CB, Boden-Albala B, Disla N, Paik MC, Elkind MS, Sacco RL. Insulin resistance and risk of ischemic stroke among nondiabetic individuals from the northern Manhattan study. Arch Neurol. 2010;67(10):1195-200.

10. Kernan WN, Inzucchi SE, Viscoli CM, Brass LM, Bravata DM, Horwitz RI. Insulin resistance and risk for stroke. Neurology. 2002;59(6):809-15.

11. Wiberg B, Sundstrom J, Zethelius B, Lind L. Insulin sensitivity measured by the euglycaemic insulin clamp and proinsulin levels as predictors of stroke in elderly men. Diabetologia. 2009;52(1):90-6.

12. Bravata DM, Wells CK, Kernan WN, Concato J, Brass LM, Gulanski BI. Association between impaired insulin sensitivity and stroke. Neuroepidemiology. 2005;25(2):69-74.

13. Kernan WN, Viscoli CM, Furie KL, Young LH, Inzucchi SE, Gorman M, Guarino PD, Lovejoy AM, Peduzzi PN, Conwit R, et al. Pioglitazone after ischemic stroke or transient ischemic attack. N Engl J Med. 2016;374(14):1321-31.

14. Lioutas VA, Alfaro-Martinez F, Bedoya F, Chung CC, Pimentel DA, Novak V. Intranasal insulin and insulin-like growth factor 1 as neuroprotectants in acute ischemic stroke. Transl Stroke Res. 2015;6(4):264-75.

15. Tam CS, Xie W, Johnson WD, Cefalu WT, Redman LM, Ravussin E. Defining insulin resistance from hyperinsulinemic-euglycemic clamps. Diabetes Care. 2012;35(7):1605-10.

16. Er LK, Wu S, Chou HH, Hsu LA, Teng MS, Sun YC, Ko YL. Triglyceride glucose-body mass index is a simple and clinically useful surrogate marker for insulin resistance in nondiabetic individuals. PLoS ONE. 2016;11(3):e0149731.

17. Ramirez-Velez R, Perez-Sousa MA, Gonzalez-Ruiz K, Cano-Gutierrez CA, Schmidt-RioValle J, Correa-Rodriguez M, Izquierdo M, Romero-Garcia JA Campos-Rodriguez AY, Triana-Reina HR, et al. Obesity- and lipid-related 
parameters in the identification of older adults with a high risk of prediabetes according to the american diabetes association: an analysis of the 2015 health, well-being, and aging study. Nutrients. 2019;11(11):2654.

18. Zhang S, Du T, Li M, Jia J, Lu H, Lin X, Yu X. Triglyceride glucose-body mass index is effective in identifying nonalcoholic fatty liver disease in nonobese subjects. Medicine (Baltimore). 2017;96(22):e7041.

19. Zheng S, Shi S, Ren X, Han T, Li Y, Chen Y, Liu W, Hou PC, Hu Y. Triglyceride glucose-waist circumference, a novel and effective predictor of diabetes in first-degree relatives of type 2 diabetes patients: cross-sectional and prospective cohort study. J Transl Med. 2016;14:260.

20. Zeng ZY, Liu SX, Xu H, Xu X, Liu XZ, Zhao XX. Association of triglyceride glucose index and its combination of obesity indices with prehypertension in lean individuals: a cross-sectional study of Chinese adults. J Clin Hypertens (Greenwich). 2020;22(6):1025-32.

21. Bala C, Gheorghe-Fronea O, Pop D, Pop C, Caloian B, Comsa H, Bozan C, Matei C, Dorobantu M. The association between six surrogate insulin resistance indexes and hypertension: a population-based study. Metab Syndr Relat Disord. 2019;17(6):328-33.

22. Sun GZ, Guo L, Wang XZ, Song HJ, Li Z, Wang J, Sun YX. Prevalence of atrial fibrillation and its risk factors in rural China: a cross-sectional study. Int J Cardiol. 2015;182:13-7.

23. Sun GZ, Li Z, Guo L, Zhou Y, Yang HM, Sun YX. High prevalence of dyslipidemia and associated risk factors among rural Chinese adults. Lipids Health Dis. 2014;13:189.

24. Xing L, Jing L, Tian Y, Liu S, Lin M, Du Z, Ren G, Sun Q, Shi L, Dai D, et al. High prevalence of stroke and uncontrolled associated risk factors are major public health challenges in rural northeast China: a populationbased study. Int J Stroke. 2019;15:399-411.

25. Du Z, Xing L, Liu S, Jing L, Tian Y, Zhang B, Yan H, Lin M, Yu S, Sun Y Prevalence and determinants of metabolic syndrome based on three definitions in rural northeast China. Public Health Nutr. 2020. https://doi. org/10.1017/S1368980019004166.

26. Hatano $\mathrm{S}$. Experience from a multicentre stroke register: a preliminary report. Bull World Health Organ. 1976;54(5):541-53.

27. Chobanian AV, Bakris GL, Black HR, Cushman WC, Green LA, Izzo JL Jr, Jones DW, Materson BJ, Oparil S, Wright JT Jr, et al. The seventh report of the joint national committee on prevention, detection, evaluation, and treatment of high blood pressure: the JNC 7 report. JAMA. 2003;289(19):2560-72.

28. Expert Panel on Detection E. Treatment of high blood cholesterol in A: executive summary of the third report of the national cholesterol education program (NCEP) expert panel on detection, evaluation, and treatment of high blood cholesterol in adults (adult treatment panel III). JAMA. 2001;285(19):2486-97.
29. Chamberlain JJ, Rhinehart AS, Shaefer CF Jr, Neuman A. Diagnosis and management of diabetes: synopsis of the 2016 American diabetes association standards of medical care in diabetes. Ann Intern Med. 2016;164(8):542-52.

30. Roden M, Price TB, Perseghin G, Petersen KF, Rothman DL, Cline GW, Shulman Gl. Mechanism of free fatty acid-induced insulin resistance in humans. J Clin Investig. 1996;97(12):2859-65.

31. Schalkwijk CG, Brouwers O, Stehouwer CD. Modulation of insulin action by advanced glycation endproducts: a new player in the field. Horm Metab Res. 2008:40(9):614-9.

32. Unoki $\mathrm{H}$, Yamagishi S. Advanced glycation end products and insulin resistance. Curr Pharm Des. 2008;14(10):987-9.

33. Shulman GI. Ectopic fat in insulin resistance, dyslipidemia, and cardiometabolic disease. N Engl J Med. 2014;371(12):1131-41.

34. Guerrero-Romero F, Simental-Mendia LE, Gonzalez-Ortiz M, MartinezAbundis E, Ramos-Zavala MG, Hernandez-Gonzalez SO, Jacques-Camarena O, Rodriguez-Moran M. The product of triglycerides and glucose, a simple measure of insulin sensitivity. Comparison with the euglycemichyperinsulinemic clamp. J Clin Endocrinol Metab. 2010;95(7):3347-51.

35. Simental-Mendia LE, Rodriguez-Moran M, Guerrero-Romero F. The product of fasting glucose and triglycerides as surrogate for identifying insulin resistance in apparently healthy subjects. Metab Syndr Relat Disord. 2008;6(4):299-304.

36. Morigny P, Houssier M, Mouisel E, Langin D. Adipocyte lipolysis and insulin resistance. Biochimie. 2016;125:259-66.

37. Kahn SE, Hull RL, Utzschneider KM. Mechanisms linking obesity to insulin resistance and type 2 diabetes. Nature. 2006;444(7121):840-6.

38. Tuo $X$, Yuan J, Wang $X H$, Xin Z. Identifying the insulin resistance index in nondiabetic Chinese subjects. Medicine (Baltimore). 2020;99(5):e19023.

39. Lim J, Kim J, Koo SH, Kwon GC. Comparison of triglyceride glucose index, and related parameters to predict insulin resistance in Korean adults: an analysis of the 2007-2010 Korean National Health and Nutrition Examination Survey. PLoS ONE. 2019;14(3):e0212963.

40. Jewell ES, Maile MD, Engoren M, Elliott M. Net reclassification improvement. Anesth Analg. 2016;122(3):818-24.

41. Michael KM, Shaughnessy M. Stroke prevention and management in older adults. J Cardiovasc Nurs. 2006;21 (5 Suppl 1):S21-26.

\section{Publisher's Note}

Springer Nature remains neutral with regard to jurisdictional claims in published maps and institutional affiliations.
Ready to submit your research? Choose BMC and benefit from:

- fast, convenient online submission

- thorough peer review by experienced researchers in your field

- rapid publication on acceptance

- support for research data, including large and complex data types

- gold Open Access which fosters wider collaboration and increased citations

- maximum visibility for your research: over 100M website views per year

At BMC, research is always in progress.

Learn more biomedcentral.com/submissions 